\title{
Estudio ecológico sobre insuficiencia renal crónica y arsénico en las aguas para consumo humano por distritos en Guanacaste
}

Ecological study on chronic kidney disease and arsenic in drinking water in districts of Guanacaste

\section{Darner Mora-Alvarado ${ }^{1}$, Azucena Urbina-Campos², Horacio Chamizo- García $^{3}$}

Fecha de recepción: 21 de octubre del 2014

Fecha de aprobación: 3 de febrero del 2015

Mora-Alvarado, D; Urbina-Campos, A; Chamizo-García, H.

Estudio ecológico sobre insuficiencia renal crónica y arsénico en las aguas para consumo humano por distritos en Guanacaste. Tecnología en Marcha. Vol. 28, № 2, Abril-Junio. Pág 102-115.

1 Microbiólogo y Máster en Salud Pública. Director del Laboratorio Nacional de Aguas del Instituto Costarricense de Acueductos y Alcantarillados (AyA). Costa Rica. Correo electrónico: dmora@aya.go.cr

2 Licenciada en Química. Funcionaria del Laboratorio Nacional de Aguas del Instituto Costarricense de Acueductos y Alcantarillados (AyA). Costa Rica.

3 Máster en Salud Pública. Catedrático de la Universidad de Costa Rica. Costa Rica. 
Tecnología en Marcha,

Vol. 28, N. ${ }^{\circ}$ 2, Abril-Junio 2015103

\section{Palabras clave}

Arsénico; agua; ecológico; insuficiencia renal; morbilidad.

\section{Resumen}

El objetivo del estudio fue analizar la asociación entre el consumo de agua contaminada con arsénico total (As-total) superior a $10 \mu \mathrm{g} / \mathrm{L}$ y los índices de morbilidad estandarizada (IME) de insuficiencia renal crónica (IRC) en los 59 distritos de los 11 cantones de la provincia de Guanacaste, Costa Rica.

Para ello se realizaron al menos 1600 muestreos y análisis de As-total en las aguas de 421 acueductos, a los cuales se les calculó el promedio ponderado según su población; luego se establecieron los IME utilizando los egresos hospitalarios de personas con esta patología. Con estas variables se diseñó un estudio ecológico-retrospectivo con características descriptivas y analíticas, aplicando la correlación lineal al 95\% de confianza entre los promedios ponderados de As-total $(\mu \mathrm{g} / \mathrm{L})$ y los IME de cada cantón. Por otro lado, se realizó un análisis de tendencia, utilizando intervalos de exposición a As-total y los IME ubicados en cada zona. Además, se elaboró un análisis descriptivo entre los 17 distritos con IME con significancia estadística y los promedios ponderados de As-total.

Como resultados, los datos de As-total indican que de los 421 acueductos, solo 11 (2,6\%) presentaron concentraciones superiores a $10 \mu \mathrm{g} / \mathrm{L}$ y pertenecen a los distritos de Cañas, Bebedero y Bagaces. Los resultados de IME de IRC indican que de los 59 distritos, 32 (54,2\%) tenían un IME mayor a 1, es decir, el riesgo de enfermar por IRC era mayor al promedio nacional en el periodo 2003-2012. Los análisis estadísticos indican una correlación simple o $r$ de 0,41, calificando la asociación entre ambas variables como Media, es decir, entre 0,33 y 0,66. El análisis de tendencias, según los intervalos o zonas de exposición a As-total, indican un comportamiento irregular en los intervalos 4 y 5, en donde la exposición "Medianamente Alta (11 a $20 \mu \mathrm{g} / \mathrm{L}$ de As-total) presenta un IME de IRC más alto que el intervalo Alta Exposición, o mayor a $20 \mu \mathrm{g} / \mathrm{L}$ de As-total. En el análisis descriptivo de los 17 distritos con IME con significancia estadística se observan varios distritos con promedios ponderados de As-total inferior a $3 \mu \mathrm{g} / \mathrm{L}$ pero con IME de IRC muy altos, como Nosara, Nacascolo, Diriá, Sámara, Palmira y Filadelfia.

Conclusiones: la comparación individual de los 17 distritos de los promedios ponderados de As-total y los IME de IRC demuestran que existen distritos que no estuvieron expuestos a As-total, pero que presentan altos IME de IRC, como Mansión, Sámara, Hojancha y Palmira. Además, se presenta la misma situación en los distritos con Muy Baja Exposición, como Liberia, Nicoya, Nacascolo, Nosara, San Antonio, Santa Cruz, Veintisiete de Abril y Diriá. Esto sugiere que el agua contaminada con As-total en los distritos de Guanacaste probablemente no es un factor de riesgo común en las zonas afectadas por la epidemia de IRC.

Recomendaciones: se debe continuar con otros estudios de tipo transversal o casos y controles, sin descartar ninguna hipótesis sobre factores de riesgo comunes en los distritos afectados con IRC, incluyendo la exposición a plaguicidas, altas temperaturas, metales pesados, incluido el As-total en aguas, y agroquímicos, entre otros.

\section{Keywords}

Arsenic; water; ecological; Chronic Kidney Disease; morbidity. 
Tecnología en Marcha,

104 Vol. 28, N. ${ }^{\circ} 2$, Abril-Junio 2015

\section{Abstract}

To analyze the association between arsenic-contaminated drinking water intake (total-As) above $10 \mathrm{ug} / \mathrm{L}$ and the Standardized Morbidity Index (SMI) for Chronic Kidney Disease (CKD) in the 59 districts of the province of Guanacaste, Costa Rica.

1600 sampling and analyses for total-As were performed on water from 421 aqueducts and population weighted averages were calculated in each of the 59 districts. SMI were established using the CKD hospital discharge data for the year 2012. Using the above variables, and through a descriptive and retrospective-analytical methodology, an ecological study was designed, in which a linear correlation was tested, (95\% confidence level), between the weighted averages (total-As, ug/L) and the SMI for the 59 districts. On the other hand a tendency analysis was performed with exposure intervals for each zone. In addition, a descriptive analysis was done for the 17 districts with a statistically significant SMI considering population weighed averages for As-total exposure.

Total-As data show that among the 421 studied aqueducts, only 17 (4,00\%) display concentrations above $10 \mathrm{ug} / \mathrm{L}$, which are located in the districts of Cañas, Bebedero, Bagaces, and La Cruz. Results for SMI for CKD indicate that among the 59 districts, 33 (55,9\%) had a SMI above "1,0", in other words, the risk of disease por CKD was over the national average for the period of study. Statistical analyses indicate a simple correlation "r" of 0,41, which can be qualified as a mediumstrength association between both variables, with a range of 0,33 and 0,66. Tendency analysis, according to the exposure intervals for As-total, show an irregular behavior within intervals 4 and 5, where the "medium-exposure" range, resulting from a weighted average between 11 and 20 ug/L, has a SMI for CKD higher than the "high-exposure" interval, which is higher than $20 \mathrm{ug} / \mathrm{L}$ total-As. In the descriptive analysis for the 17 districts with statistically significant SMI, some districts with a population-weighted average of total-As below $3 \mathrm{ug} / \mathrm{L}$, but with a very high SMI for CKD, such as Nosara, Nacascolo, Diriá, Sámara, Palmira and Filadelfia.

Conclusions: even though the tendency analysis shows there is an increased risk of illness as exposure augments up to the exposure range, individual comparison of the population-weighted averages for total-As and the SMI for CKD of the 17 districts; it is evident that some districts that were not exposed to total -As have a high SMI for CKD, such as Mansión, Sámara, Hojancha and Palmira. The same situation is also seen for the very low exposure category, such as Liberia, Nicoya, Nacascolo, Nosara, San Antonio, Santa Crur, 27 de Abril and Diriá. This suggests that total-As contaminated water, in the districts of Guanacaste, probably is not a common risk factor in the zones affected by the CKD epidemic.

Recommendations: cross sectional and case/control studies should be done, without neglecting any hypothesis regarding the common risk factors in the CKD affected districts, including exposure to pesticides, high temperature, heavy metals (including total-As in drinking water) agrochemicals, agricultural occupation, among others.

\section{Introducción}

En el año 2002 la National Kidney Foundation de Estados Unidos definió la insuficiencia renal crónica (IRC) como la "presencia de daño renal con una duración igual o mayor a tres meses, caracterizada por anormalidades estructurales o funcionales con o sin descenso de las tasas de filtración glomerular a menos de $60 \mathrm{~mL} / \mathrm{min} / 1,73$ m²" (National Kidney Fundation, 2002). La IRC es un proceso fisiopatológico multifactorial de carácter progresivo e irreversible, que con frecuencia lleva a un estado terminal al paciente, que requiere de terapia de reemplazo, diálisis o trasplante de riñón. 
La creciente incidencia y prevalencia de esta patología en países desarrollados oscila entre $13 \%$ y $16 \%$, convirtiéndose en un verdadero problema de salud pública a nivel mundial; en los países en desarrollo varía de 0\% a 16\% (Soyibo y Roberts, 2011; Calvo et al., 2010; Flores, 2009). Las causas o etiologías, de acuerdo con su fisiopatología, se clasifican en vascular, glomerular, túbulo intersticial y obstructiva e involucran al menos 80 agentes causales, de los cuales existen algunos de iniciación, de susceptibilidad porque aumentan el daño renal, y otros de progresión causantes del deterioro de los riñones (Brooks, 2009). Los principales agentes etiológicos son diabetes, hipertensión arterial, glomerulonefritis, enfermedades poliquísticas y cálculos renales (Ministerio de Salud de Chile, 2010).

No obstante, en algunos países como Sri-Lanka, Egipto, India, México y en la costa pacífica de Centroamérica se ha observado, en las últimas dos décadas, un crecimiento de la prevalencia de IRC de etiología desconocida que no cumple con los factores de riesgo tradicionales (Wanigasuriya et al., 2014; Saraj, 2014; Xavier, 2012; López et al., 2014). Por ejemplo, en Nicaragua los estudios de prevalencia varían de $0 \%$ a 13\%, con una media de 8,7\%; la mortalidad en el periodo de 1992 a 2005 pasó de 4,5 a 10,9/100.000 habitantes y los departamentos más afectados han sido León y Chinandega, en la costa pacífica, que presentan actividades comerciales comunes como cultivo de caña, algodón y el uso de al menos 20 tipos de agroquímicos, algunos de los cuales son nefrotóxicos (Ramírez, 2011; Callejas et al., 2003). Lo mismo sucede en el Salvador, en donde otro factor de riesgo es la alta temperatura a que se exponen los trabajadores agrícolas (Henneva et al., 2014; Rodríguez, 2014; Almaguer et al., 2014).

Ante esto, en el año 2009 la Escuela de Salud Pública de Boston (EE. UU.) inició investigaciones para determinar las causas de la epidemia. Primero se realizaron 22 estudios, en los que se evaluaron exposiciones a situaciones como el trabajo agrícola (caña de azúcar, algodón, arroz, banano y café), metales pesados en aguas, suelos, plaguicidas, condiciones médicas incluyendo la deshidratación, infecciones del tracto urinario (chistate), diabetes, hipertensión, uso de antiinflamatorios, consumo de alcohol y lija (ron), tabaquismo e historial familiar de IRC. De estas posibles causas, en forma consistente se observaron asociaciones positivas para el trabajo agrícola en ingenios o cultivos de caña de azúcar, exposición a plaguicidas, deshidratación, hipertensión, consumo de lija e historial familiar con IRC (Cuadra et al., 2005).

En Costa Rica también se ha observado un incremento de los casos de IRC. En la provincia de Guanacaste, también ubicada en la costa pacífica, la incidencia ha sido de 112,9/100.000 habitantes, triplicando el promedio nacional (Blanco, 2013). Aunque las condiciones laborales y el clima son semejantes a los de las zonas de El Salvador, Nicaragua y otros países costeros de Mesoamérica, las fuerzas vivas y algunos políticos, en conjunto con varios medios de comunicación, han vinculado directamente la IRC con el consumo de agua contaminada con trazas de arsénico total (As-total) superiores a $10 \mu \mathrm{g} / \mathrm{L}$, tipificado en el Reglamento para la Calidad del Agua Potable (Poder Ejecutivo de Costa Rica, 2005). Este vínculo ha sido generalizado para la totalidad de la provincia de Guanacaste, cuando en realidad los distritos expuestos a concentraciones superiores a $10 \mu \mathrm{g} / \mathrm{L}$ de As-total son Bagaces, Cañas y Bebedero (Mora, 2011; Mora, 2013; Mora, 2014) (Grupo de Trabajo AyA y Comisión Agua Segura MINSA, 2013). En razón de esta situación se realiza el presente estudio, con el objetivo de analizar la asociación entre el consumo de agua contaminada con As-total superior a $10 \mu \mathrm{g} / \mathrm{L}$ y los índices de morbilidad estandarizado (IME) de IRC en los 59 distritos de los 11 cantones de la provincia de Guanacaste. 
Tecnología en Marcha,

106 Vol. 28, N. ${ }^{\circ} 2$, Abril-Junio 2015

\section{Metodología}

Para cumplir con el objetivo del estudio se definieron tres etapas. En la primera se efectuaron los muestreos y análisis de As-total de los 421 acueductos inventariados en los 59 distritos estudiados. Con los datos de As-total se calcularon los promedios ponderados, de acuerdo con la población de cada distrito. Los muestreos y los análisis de aguas se ejecutaron con procedimientos acreditados con la Norma INTE-ISO/IEC 17025: 2005, del Laboratorio Nacional de Aguas (LNA), en el periodo comprendido entre septiembre de 2009 y julio de 2014.

Los muestreos se realizaron en 43 acueductos operados por el Instituto Costarricense de Acueductos y Alcantarillados (AyA), dos sistemas municipales y 376 acueductos comunales a cargo de Asociaciones Administradoras de Acueductos (Asadas) y/o Comités Administradores de Acueductos Rurales (CAARs).

Las técnicas utilizadas en la cuantificación de las concentraciones de As-total fueron:

a) Absorción atómica con horno de grafito

b) Espectrometría de masas con fuente de plasma de acoplamiento inductivo (ICP-MASAS).

Ambas técnicas cumplen con las directrices del Standard Methods for Examination of Water and Waste Water Method, 53 B. 22 edition (APHS/AWWA/WEF, 2012).

Luego, para el cálculo de los promedios ponderados de As-total ( $\mu \mathrm{g} / \mathrm{L})$ en los acueductos de cada distrito, se aplicó la siguiente fórmula:

$$
\sum \frac{W_{i}}{W_{t}}(\bar{X})
$$

$W_{i}$ : Población que abastece cada acueducto

$W_{t}$ : Población total por distrito con datos de exposición a As

$\bar{X}$ : Promedio aritmético de la concentración de As en cada acueducto.

En la segunda etapa se calculó el IME de IRC para cada uno de los 459 distritos del país, y posteriormente en forma específica para los 59 distritos de la provincia de Guanacaste.

Los egresos hospitalarios se obtuvieron del área de estadística de la Caja Costarricense de Seguro Social (CCSS) y la población del Centro Centroamericano de Población (CCP) (CCP, 2012), para el periodo comprendido entre los años 2003 y 2012. Con estos datos de egresos hospitalarios por IRC se calculó la morbilidad bruta por 1000 personas, la cual corresponde al cociente del total de casos ocurridos entre el total de la población de cada distrito. Luego, se determinaron los IME de IRC como indicador de riesgo para grupos de edades quinquenales, lo cual refleja la probabilidad de enfermar contemplando el efecto de la edad. Para calcular los IME se empleó un método indirecto, que ha sido utilizado en investigaciones similares (Benach et al., 1998) así como por la disponibilidad y accesibilidad para descargar la información de las bases de datos disponibles en línea. Con los datos disponibles se realizó un análisis espacial de los últimos 10 años, mediante mapas de la división política administrativa de distritos del Atlas del Instituto Tecnológico de Costa Rica (ITCR), tanto para el país como para la provincia de Guanacaste (Instituto Tecnológico de Costa Rica, 2008).

Una vez realizados los cálculos de IME de IRC de los 59 distritos guanacastecos, se procedió a identificar los que obtuvieron valores superior a 1, y entre estos los que tenían significado estadístico. El valor de IME de 1 refleja el promedio nacional del riesgo de enfermar de IRC en cada distrito. 
La tercera etapa se dividió en tres fases. En la primera se sometieron las variables de los promedios ponderados de As-total y los IME de IRC por distritos a un análisis de correlación lineal al 95\% de confianza, en los 59 distritos. En la segunda se elaboró un análisis de exposición por intervalos de los promedios ponderados de As-total e IME de IRC correspondiente. Los cinco intervalos se definieron de la siguiente forma:

Intervalo 1 (No expuesto): el promedio ponderado de As-total en las aguas para consumo humano $(\mathrm{ACH})$ por distritos que arrojaron resultados de ND (No Detectado), los cuales, para efectos prácticos, definimos con el valor de 1 y no de 0 , debido a que en la cuantificación de análisis químicos no existe el cero.

Intervalo 2 (Muy baja exposición): el promedio ponderado de As-total en los distritos con valores entre $>1-3 \mu \mathrm{g} / \mathrm{L}$. Este intervalo se identificará como D (Detectable pero no cuantificable).

Intervalo 3 (Baja exposición): el promedio ponderado de As-total de los distritos con resultados entre $>3-10 \mu \mathrm{g} / \mathrm{L}$.

Intervalo 4 (Mediana exposición): el promedio ponderado de As-total de los distritos con resultados entre $>10-20 \mu \mathrm{g} / \mathrm{L}$.

Intervalo 5 (Alta exposición): el promedio ponderado de As-total de los distritos con resultados $>20 \mu \mathrm{g} / \mathrm{L}$.

En cada intervalo se ubican los IME de IRC de la zona correspondiente. Luego, los resultados se visualizan a través de un gráfico para analizar si existe alguna tendencia entre los intervalos de As-total y los IME de IRC.

Por último, en la tercera fase, y fundamentados en el principio epidemiológico "de que en zonas afectadas por una enfermedad de origen desconocido, se deben buscar factores de riesgo comunes para definir la causalidad de la misma", se graficaron los datos promedio de As-total y el IME de IRC de cada distrito, en donde el IME tenía significancia estadística al 95\% de confianza.

\section{Resultados}

Los resultados de esta investigación se presentan, en concordancia con La metodología, en tres etapas.

El total de los 421 acueductos de la provincia de Guanacaste, evaluados en el periodo de septiembre de 2009 al 15 de julio de 2014, se distribuyen en ND (No detectado), D (Detectado pero no cuantificable), de >3-10 $\mu \mathrm{g} / \mathrm{L},>10-20 \mu \mathrm{g} / \mathrm{L}$ y $>20 \mu \mathrm{g} / \mathrm{L}$ de As-total.

El resumen, los análisis de As-total en las aguas para consumo se distribuyen de la siguiente manera: 1.042 (64,1\%) ND, 33 (2,03\%) D, 349 (21,5\%) de >3-10 $\mu \mathrm{g} / \mathrm{L}, 93$ (5,72) >10-20 $\mu \mathrm{g} / \mathrm{L}, y$ $109(6,7 \%)>20 \mu \mathrm{g} / \mathrm{L}$, para un total de 1626 análisis de As-total en los 59 distritos guanacastecos.

En el cuadro 1 se resumen los datos de los promedios ponderados de As-total en las aguas para consumo con IME de IRC en los 59 distritos de Guanacaste. Además, se presenta la morbilidad bruta de los límites inferiores y superiores de IRC. Por otro lado, mediante un asterisco, se identifican los distritos que presentan el IME de IRC con significancia estadística al 95\% de confianza. 
Cuadro 1. Guanacaste: promedios ponderados de As-total e Índice de Morbilidad Estandarizado por Edad por distritos en Guanacaste

\begin{tabular}{|c|c|c|c|c|c|}
\hline $\begin{array}{c}\text { Código y nombre de distritos de } \\
\text { la provincia }\end{array}$ & $\begin{array}{l}\text { Promedio } \\
\text { ponderado de } \\
\text { As-total } \mu / L\end{array}$ & $\begin{array}{l}\text { Índice de Morbilidad } \\
\text { Estandarizado por Edad } \\
\text { de Insuficiencia Renal }\end{array}$ & $\begin{array}{c}\text { IC } \\
\text { inferior }\end{array}$ & $\begin{array}{c}\text { IC } \\
\text { superior }\end{array}$ & $\begin{array}{l}\text { Morbilidad } \\
\text { bruta }\end{array}$ \\
\hline 50101: Liberia* & 1,59 & 2,25 & 1,70 & 2,81 & 1,40 \\
\hline 50102: Cañas Dulces & 1,35 & 1,16 & 0,00 & 2,76 & 0,79 \\
\hline 50103: Mayorga & 1,32 & 1,19 & 0,00 & 3,51 & 0,75 \\
\hline 50104: Nacascolo* & 2,31 & 8,25 & 2,86 & 13,64 & 5,33 \\
\hline 50105: Curubandé & 1,63 & 2,92 & 0,06 & 5,77 & 1,85 \\
\hline 50201: Nicoya* & 2,69 & 3,63 & 2,67 & 4,59 & 2,66 \\
\hline 50202: Mansión* & 1,00 & 3,08 & 1,26 & 4,90 & 2,51 \\
\hline 50203: San Antonio* & 1,76 & 3,16 & 1,70 & 4,62 & 2,81 \\
\hline 50204: Quebrada Honda & 1,12 & 2,78 & 0,34 & 5,22 & 2,23 \\
\hline 50205: Sámara* & 1,00 & 6,88 & 3,14 & 10,61 & 4,53 \\
\hline 50206: Nosara* & 1,84 & 12,50 & 7,79 & 17,22 & 8,07 \\
\hline 50207: Belén de Nosarita & 2,28 & 0,64 & 0,00 & 1,89 & 0,48 \\
\hline 50301: Santa Cruz & 1,22 & 3,76 & 2,71 & 4,80 & 2,75 \\
\hline 50302: Bolsón & 1,00 & 3,17 & 0,06 & 6,29 & 2,51 \\
\hline 50303: Veintisiete de Abril* & 1,03 & 3,45 & 1,57 & 5,32 & 2,60 \\
\hline 50304: Tempate & 1,23 & 3,01 & 0,92 & 5,09 & 2,15 \\
\hline 50305: Cartagena & 1,65 & 2,07 & 0,26 & 3,89 & 1,42 \\
\hline 50306: Cuajiniquil & 1,87 & 2,50 & 0,00 & 5,97 & 1,72 \\
\hline 50307: Diriá* & 1,10 & 6,14 & 3,22 & 9,05 & 5,21 \\
\hline 50308: Cabo Velas & 1,00 & 2,06 & 0,04 & 4,09 & 1,27 \\
\hline 50309: Tamarindo & 1,15 & 0,72 & 0,00 & 1,73 & 0,49 \\
\hline 50401: Bagaces* & 32,04 & 6,29 & 4,39 & 8,20 & 4,07 \\
\hline 50402: Fortuna & 1,21 & 0,00 & 0,00 & 0,00 & 0,00 \\
\hline 50403: Mogote & 1,11 & 0,00 & 0,00 & 0,00 & 0,00 \\
\hline 50404: Río Naranjo & 1,00 & 0,00 & 0,00 & 0,00 & 0,00 \\
\hline 50501: Filadelfia* & 3,15 & 5,03 & 3,02 & 7,04 & 3,25 \\
\hline 50502: Palmira* & 2,99 & 5,19 & 2,47 & 7,90 & 3,31 \\
\hline 50503: Sardinal & 1,08 & 0,93 & 0,24 & 1,62 & 0,58 \\
\hline 50504: Belén & 1,00 & 1,41 & 0,28 & 2,55 & 0,97 \\
\hline 50601: Cañas* & 25,10 & 5,57 & 4,24 & 6,90 & 3,43 \\
\hline 50602: Palmira & 1,00 & 0,00 & 0,00 & 0,00 & 0,00 \\
\hline
\end{tabular}


Continuación

\begin{tabular}{|c|c|c|c|c|c|}
\hline $\begin{array}{c}\text { Código y nombre de distritos de } \\
\text { la provincia }\end{array}$ & $\begin{array}{c}\text { Promedio } \\
\text { ponderado de } \\
\text { As-total } \mu / L\end{array}$ & $\begin{array}{l}\text { Índice de Morbilidad } \\
\text { Estandarizado por Edad } \\
\text { de Insuficiencia Renal }\end{array}$ & $\begin{array}{c}\text { IC } \\
\text { inferior }\end{array}$ & $\begin{array}{c}\text { IC } \\
\text { superior }\end{array}$ & $\begin{array}{c}\text { Morbilidad } \\
\text { bruta }\end{array}$ \\
\hline 50603: San Miguel & 1,00 & 1,05 & 0,00 & 3,10 & 0,78 \\
\hline 50604: Bebedero* & 15,13 & 10,41 & 5,31 & 15,52 & 6,11 \\
\hline 50605: Porozal & 1,71 & 0,00 & 0,00 & 0,00 & 0,00 \\
\hline 50701: Las Juntas & 1,46 & 1,15 & 0,30 & 2,00 & 0,79 \\
\hline 50702: Sierra & 1,22 & 0,63 & 0,00 & 1,88 & 0,39 \\
\hline 50703: San Juan & 1,23 & 0,00 & 0,00 & 0,00 & 0,00 \\
\hline 50704: Colorado & 2,30 & 0,36 & 0,00 & 1,08 & 0,23 \\
\hline 50801: Tilarán & 1,00 & 0,55 & 0,00 & 1,18 & 0,40 \\
\hline 50802: Queb Grande & 1,41 & 2,35 & 0,05 & 4,64 & 1,53 \\
\hline 50803: Tronadora & 1,27 & 0,00 & 0,00 & 0,00 & 0,00 \\
\hline 50804: Santa Rosa & 1,87 & 0,00 & 0,00 & 0,00 & 0,00 \\
\hline 50805: Líbano & 1,06 & 1,56 & 0,00 & 4,61 & 1,34 \\
\hline 50806: Tierras Morenas & 1,06 & 0,00 & 0,00 & 0,00 & 0,00 \\
\hline 50807: Arenal & 1,61 & 0,00 & 0,00 & 0,00 & 0,00 \\
\hline 50901: Carmona & 1,00 & 0,65 & 0,00 & 1,93 & 0,49 \\
\hline 50902: Santa Rita & 1,00 & 0,94 & 0,00 & 2,79 & 0,67 \\
\hline 50903: Zapotal & 1,00 & 2,98 & 0,00 & 6,34 & 2,49 \\
\hline 50904: San Pablo & 1,00 & 0,00 & 0,00 & 0,00 & 0,00 \\
\hline 50905: Porvenir & 1,00 & 0,00 & 0,00 & 0,00 & 0,00 \\
\hline 50906: Bejuco & 1,00 & 0,57 & 0,00 & 1,69 & 0,40 \\
\hline 51001: La Cruz* & 3,62 & 2,61 & 1,19 & 4,02 & 1,60 \\
\hline 51002: Santa Cecilia & 1,00 & 1,58 & 0,20 & 2,96 & 0,88 \\
\hline 51003: La Garita & 1,73 & 0,00 & 0,00 & 0,00 & 0,00 \\
\hline 51004: Santa Elena & 2,00 & 0,00 & 0,00 & 0,00 & 0,00 \\
\hline *51101: Hojancha & 1,00 & 4,36 & 1,89 & 6,83 & 3,24 \\
\hline 51102: Monte Romo & 1,00 & 0,00 & 0,00 & 0,00 & 0,00 \\
\hline 51103: Puerto Carrillo & 1,00 & 0,00 & 0,00 & 0,00 & 0,00 \\
\hline 51104: Huacas & 1,00 & 0,00 & 0,00 & 0,00 & 0,00 \\
\hline
\end{tabular}

Fuente: Elaborado por los autores.

* Distritos con IME de IRC con significancia estadística al 95\% de confianza.

En el mismo cuadro 1 se observan los 33 distritos (55,9\%) con IME de IRC superior a 1 o al promedio nacional, lo cual significa mayor riesgo de enfermar por dicha enfermedad.

En las figuras 1 y 2 se ubican, por intervalos, los IME de IRC en 459 distritos de Costa Rica y los 59 distritos de Guanacaste, respectivamente. 
Índice de Mortalidad Estandarizado por

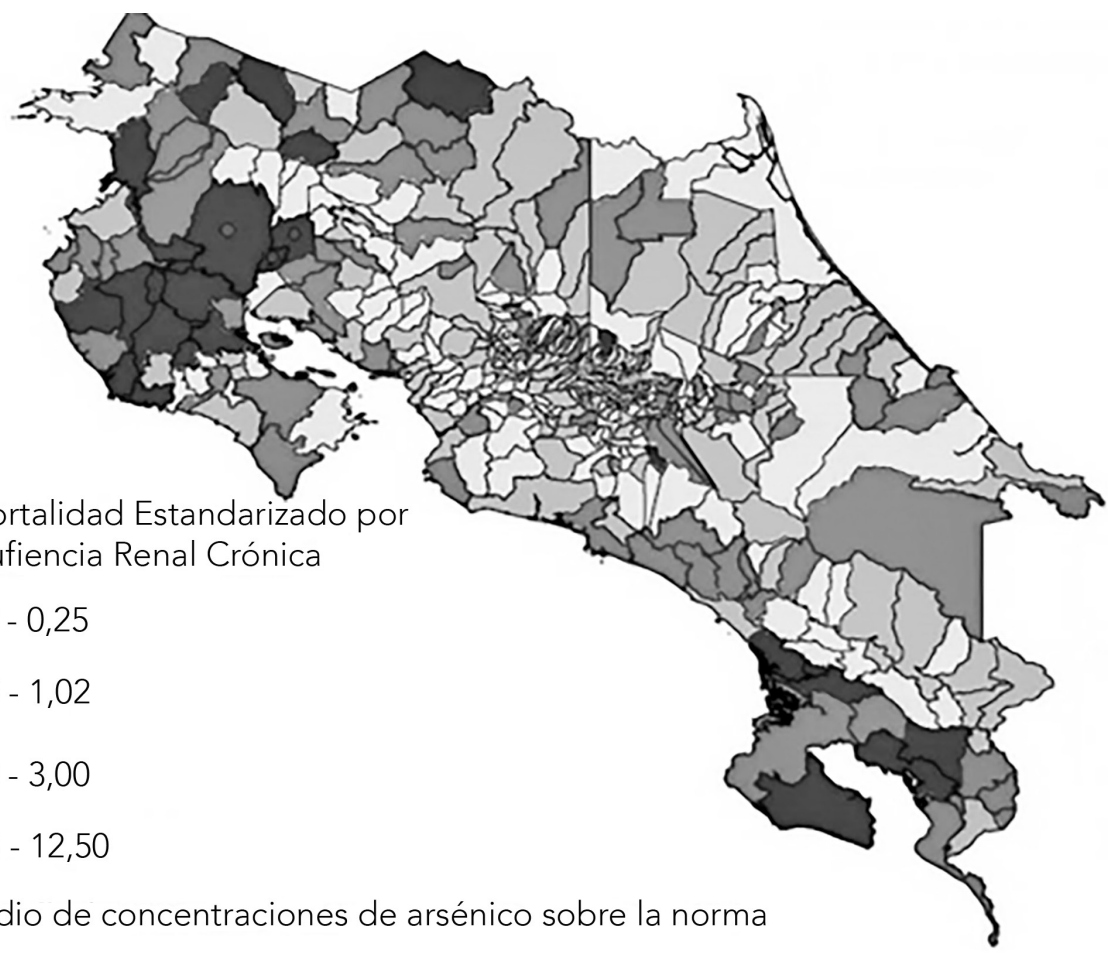

Figura 1. Índice de Morbilidad Estandarizado por Edad de Insuficiencia Renal Crónica de 2003 a 2012 en Costa Rica y promedios de concentraciones de As sobre la norma

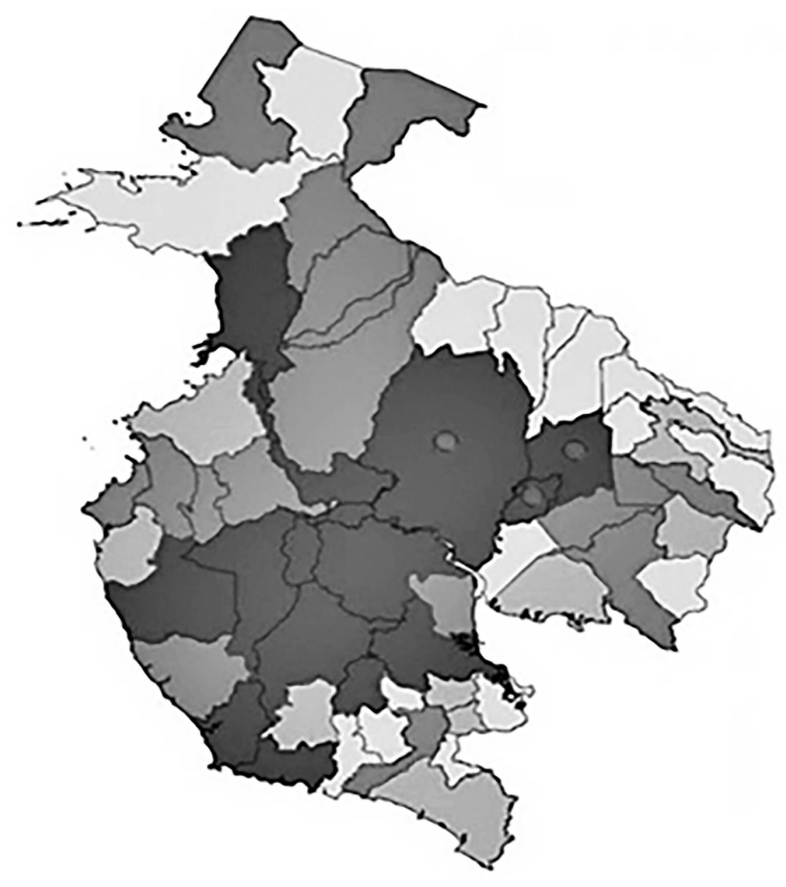

Índice de Mortalidad Estandarizado por Edad de insufiencia Renal Crónica

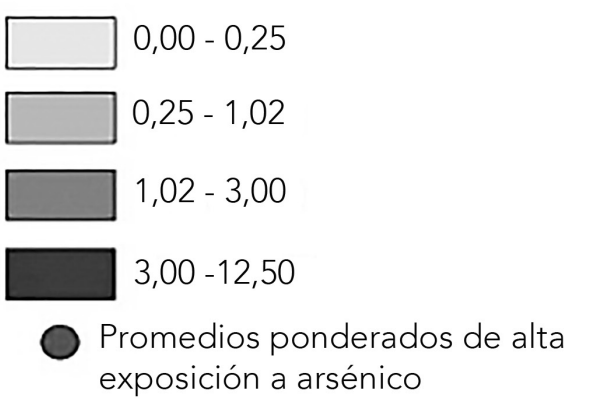

Figura 2. Índice de Morbilidad Estandarizado por Edad de Insuficiencia Renal Crónica de 2003 a 2012 en Guanacaste y promedios ponderados de alta exposición a As 
El análisis de correlación lineal, al 95\% de confianza, de los pares de promedios ponderados de As-total $(\mu \mathrm{g} / \mathrm{L})$ y los IME de IRC, indica un resultado de 0,41, lo cual refleja una asociación media $(0,33$ a 0,66$)$ entre ambas variables.

Con respecto a las zonas de exposición a As, según promedios ponderados por población e insuficiencia renal crónica al año 2012 en Guanacaste, se utilizó el código de la décima Clasificación Internacional de Enfermedades CIE-10 N18.

Los resultados obtenidos con esta clasificación, según los promedios de As-total ponderados en cada población, son:

Intervalo 1 (No Expuesto): Mansión, Sámara, Bolsón, Cabo Velas, Río Naranjo, Belén, Palmira, San Miguel, Tilarán, Carmona, Santa Rita, Zapotal, San Pablo, Porvenir, Bejuco, Santa Cecilia, Hojancha, Monte Romo, Puerto Carrillo y Huacas.

Intervalo 2 (Muy baja exposición): Liberia, Cañas Dulces, Mayorga, Nacascolo, Curubandé, Nicoya, San Antonio, Quebrada Honda, Nosara, Belén de Nosarita, Santa Cruz, Veintisiete de Abril, Tempate, Cartagena, Cuajiniquil, Diriá, Tamarindo, Fortuna, Mogote, Palmira, Sardinal, Porosal, Juntas, Sierra, San Juan, Colorado, Quebrada Grande, Tronadora, Santa Rosa, Líbano, Tierras Morenas, Arenal, La Garita y Santa Elena.

Intervalo 3 (Baja exposición): Filadelfia y La Cruz.

Intervalo 4 (Mediana exposición): Bebedero.

Intervalo 5 (Alta exposición): Bagaces y Cañas.

En la figura 3 se observa el IME por edad de cada zona; el caso de mediana exposición es el distrito de Bebedero.

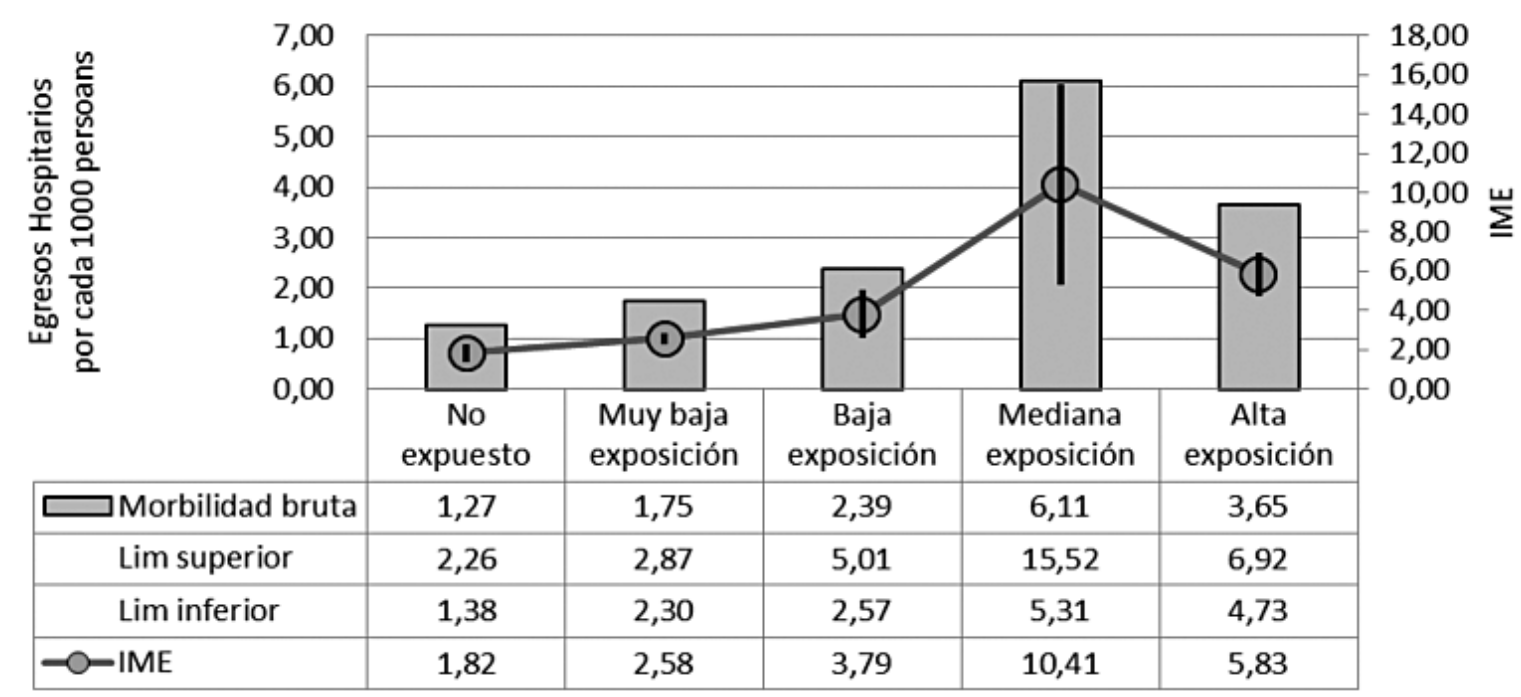

Figura 3. Morbilidad de insuficiencia renal crónica por zonas de exposición a arsénico en Guanacaste por distritos de 2003 a 2012.

De los 59 distritos de Guanacaste, se seleccionaron los 17 que tenían IME de IRC superior a 1 y con significancia estadística y se compararon con los promedios de As-total respectivos. Dichos resultados se observan en la figura 4. 


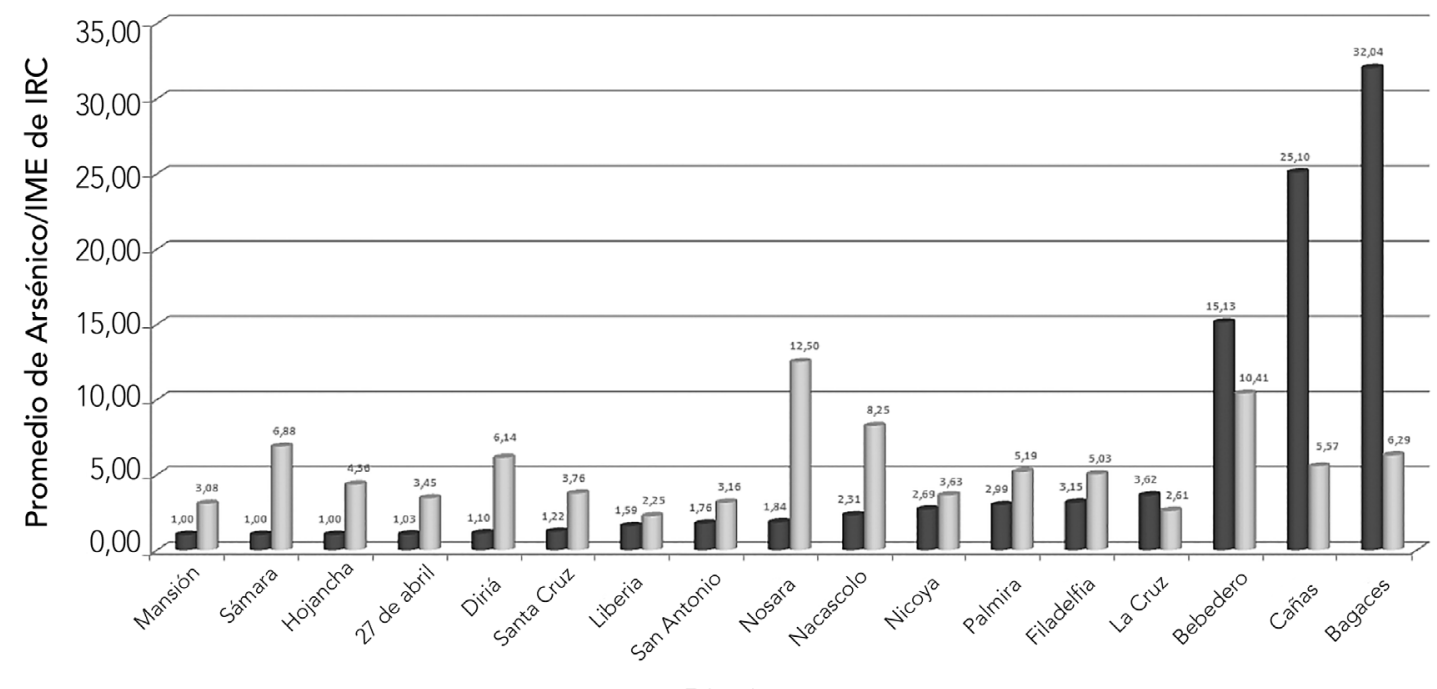

Distrito

uPromedio As $\square$ IME

Fuente Laboratorio Nacional de Aguas

Figura 4. Promedios Ponderados de As-total y IME de IRC con Significancia Estadística en 17 Distritos de Guanacaste.

\section{Análisis de resultados}

De conformidad con el orden establecido en la presentación de los resultados, a continuación se realiza el análisis de las mismas tres etapas:

Con respecto a la primera etapa, los exámenes de As-total en los 421 acueductos distribuidos en los 59 distritos, el 87,5\% presentaron resultados inferiores a $10 \mu \mathrm{g} / \mathrm{L}$; de ellos, el 73,2\% mostró concentraciones ND, el 2,3\% resultados D y el $24,5 \%$ valores en el intervalo de $>3-10$ $\mu \mathrm{g} / \mathrm{L}$.

El $12,5 \%$ de los resultados mayores a $10 \mu \mathrm{g} / \mathrm{L}$ se concentró en los distritos de Bagaces, Cañas y Bebedero de Cañas. En el caso de Cañas y Bagaces, los datos utilizados fueron los determinados antes de las soluciones aplicadas por el AyA en los respectivos acueductos.

Con el propósito de obtener datos de As-total lo más cercanos posible a la realidad de cada distrito, se aplicó el cálculo de los promedios ponderados por población, utilizando la fórmula mencionada en la metodología. Los resultados indican que:

- De los 59 distritos, 3 (5,1\%) tenían resultados mayores a $10 \mu \mathrm{g} / \mathrm{L}$ de As-total; estos fueron Bagaces $(32,04 \mu \mathrm{g} / \mathrm{L})$, Cañas $(25,10 \mu \mathrm{g} / \mathrm{L})$ y Bebedero $(15,13 \mu \mathrm{g} / \mathrm{L})$.

- Dos distritos $(3,4 \%)$ se ubicaron en el intervalo de $>3-10 \mu \mathrm{g} / \mathrm{L}$; estos fueron: Filadelfia $(3,15$ $\mu \mathrm{g} / \mathrm{L})$ y La Cruz $(3,62 \mu \mathrm{g} / \mathrm{L})$.

- Un total de 34 distritos $(57,6 \%)$ se ubicaron en el intervalo >1-3 $\mu \mathrm{g} / \mathrm{L}$ de As-total.

- 20 distritos presentaron un promedio ponderado de 1, para un 33,9\%.

En la segunda etapa se determina que los IME de IRC por distritos, calculados a partir de los egresos hospitalarios observados entre los esperados, indican que: 
- De los 59 distritos, 33 (55,9\%) tenían resultados superiores a 1 o al promedio nacional; es decir, el riesgo de enfermar por IRC es mayor que en distritos con IME igual o menor que 1.

- De los 33 distritos con IME de IRC superior a 1, un total de 17 (51,5\%) tenían IME con significancia estadística.

Por otro lado, la figura 1 permite observar conglomerados de IRC en la provincia de Guanacaste. En la figura 2 se aprecia que la epidemia de IRC, de origen desconocido, se concentra en 17 distritos (con significancia estadística) incluidos Mansión, Sámara, Hojancha, Veintisiete de Abril, Diriá, Santa Cruz, Liberia, San Antonio, Nosara, Nacascolo, Nicoya, Palmira, Filadelfia, La Cruz, Bebedero, Cañas y Bagaces.

En la primera fase de la tercera etapa, el análisis de correlación lineal al 95\% de confianza, entre los datos de As-total y los IME de IRC en los 59 distritos de Guanacaste, indican un valor "r" de 0,41, el cual demuestra una asociación media entre un valor de ("r" 0,33 a 0,66) entre ambas variables.

Con respecto a la segunda fase de la clasificación por zonas de exposición a As-total, en la figura 3 no se observa una tendencia concordante, que indique que a mayor promedio de As-total mayor es el incremento de IME de IRC entre los cinco intervalos, sobre todo entre los de baja, mediana y alta exposición.

En la tercera fase, sobre las concentraciones promedio de As-total y los IME de IRC para cada uno de los 17 distritos con significancia estadística, en la figura 4 se observa que:

Bagaces, Cañas y Bebedero tienen IME de IRC de 6,29, 5,57, y 10,41 con As-total promedio de $32,04 \mu \mathrm{g} / \mathrm{L}, 25,10 \mu \mathrm{g} / \mathrm{L}$ y $15,13 \mu \mathrm{g} / \mathrm{L}$, respectivamente.

La comparación individual de los promedios ponderados de As-total y los IME de IRC de los 17 distritos demuestra que existen algunos que no estuvieron expuestos a As-total pero que presentan altos IME de IRC, como Mansión, Sámara, Hojancha y Palmira. Además, se presenta la misma situación en los distritos con muy baja exposición, como Liberia, Nicoya, Nacascolo, Nosara, San Antonio, Santa Cruz, Veintisiete de Abril y Diriá. Esto sugiere que el agua contaminada con As-total en los distritos de Guanacaste no es el factor de riesgo inicial de la epidemia de IRC.

\section{Conclusiones y recomendaciones}

El análisis de los resultados permite presentar las siguientes conclusiones y recomendaciones.

\section{Conclusiones}

La distribución de los exámenes de As-total en las ACH suplidas por acueductos demuestran que solo tres distritos guanacastecos están expuestos a As-total mayor a $10 \mu \mathrm{g} / \mathrm{L}(5,1 \%)$; es decir, los 56 distritos restantes $(94,9 \%)$ tienen valores inferiores a $10 \mu \mathrm{g} / \mathrm{L}$, que es el valor permitido por el Reglamento para la Calidad del Agua Potable.

Los resultados de las tres fases -las estadísticas de análisis de correlación lineal al 95\% de confianza de un $r$ de 0,41, el análisis de tendencias de exposición a As-total e IME de IRC respectivo y el estudio descriptivo de los promedios ponderados de As-total y los IME de IRC en los 17 distritos con significancia estadística permiten concluir que:

- No se observa una correlación lineal fuerte entre ambas variables de As-total e IME de IRC en los 59 distritos de Guanacaste. 
- No se observa concordancia entre la exposición a As-total, en los intervalos de Mediana exposición y Alta exposición, y los IME de IRC en las respectivas zonas, es decir, es mayor el IME de IRC entre 11-20 $\mu \mathrm{g} / \mathrm{L}$ que en $>20 \mu \mathrm{g} / \mathrm{L}$.

- La comparación individual de los 17 distritos, de los promedios ponderados de As-total y los IME de IRC demuestra que existen distritos que no estuvieron expuestos a As-total pero presentan IME de IRC altos, como Mansión, Sámara, Hojancha y Palmira. Además, se presenta la misma situación en los distritos con muy baja exposición, como Liberia, Nicoya, Nacascolo, Nosara, San Antonio, Santa Cruz, Veintisiete de Abril y Diriá. Esto sugiere que el agua contaminada con As-total en los distritos de Guanacaste probablemente no es un factor de riesgo común en las zonas afectadas por la epidemia de IRC.

\section{Recomendaciones}

Con el propósito de detectar las verdaderas causas de la IRC en Guanacaste y otras comunidades de la costa pacífica de Mesoamérica, es fundamental aplicar el principio de buscar factores de riesgo comunes en las diferentes zonas afectadas, como el tipo de trabajo, la exposición a plaguicidas y las altas temperaturas durante la jornada laboral, litiasis renal y uso de antiinflamatorios, entre otros.

Por último, es necesario tomar medidas urgentes para atender esta epidemia de IRC de origen desconocido, en el marco de la "Declaración de San Salvador" de la Comisión de Ministerios de Salud de Centroamérica y República Dominicana (COMISCA) y el $52^{\circ}$ Consejo Directivo $65^{a}$ Sesión del Comité Regional (OMS/OPS), del 4 de septiembre de 2013. (OMS/OPS, 2013).

\section{Bibliografía}

Almaguer, M., Herrera, R. \& Orontes, C. (abril, 2014). Chronic Kidney Disease of Unknown Etiology in Agricultural Communities. Medicc Review 16(2).

APHS/AWWA/WEF. (2012). Standard Methods for the Examination of Water and Waste Water. 22 ed. Washington D.C.

Ariana Ramírez Rubio, Madeleine Kangsen Scammell. Enfermed Renal Crónica en Nicaragua. Análisis Cualitativo de Entrevistas Semiestructurales con Médicos y Farmacéuticos. Universidad de Boston; 2011.

Benach, J., Borrell, C., García, M. D. \& Chamizo, H. (1998). Desigualdades sociales en mortalidad en áreas pequeñas de España SESPAS. Informe 1998. Granada: Sociedad Española de Salud Pública y Administración Sanitaria.

Blanco, C. (19 agosto, 2013). Químicos causan insuficiencia renal crónica y muerte en Guanacaste. La Prensa Libre, sección Enfoque.=

Brooks, D. (2009). Informe Final de Estudio de Alcance: Epidemiología de Enfermedad Renal en Nicaragua. Informe Independiente Preparado por la Escuela de Salud Pública de la Universidad de Boston.

Callejas Callejas, Li Medrano Alonso, C. D; Mendoza. (2003). Insuficiencia Renal Crónica (IRC) en trabajadores de caña de azúcar. El Viejo, Chinandega. Managua: Centro para el Control y Prevenciones de Enfermedad (CDC) de Estados Unidos,

Ministerio de Salud de Nicaragua. Calvo, M. et al. (2010). Evaluación de la comparación corporal en pacientes con Insuficiencia Renal Crónica. Nutr. blosp., 25(4), 682-687.

Centro Centroamericano de Población (CCP) (2014). Datos en línea e UCR. Información demográfica. San José: Universidad de Costa Rica. Obtenido de www.ccc.ac.cr.

Cuadra S. N., Jakobsson, K. \& Wessling, C. (2006). Chronic renal disease in Central America. An assessment of avaible information. SALTRA report 2005/6.

Flores, J. (2010). Enfermedad Renal Crónica: Epidemiología y Factores de Riesgo. Rev. Med. Clin. Condes, 2(4), 502-507. 
Grupo de trabajo sobre Arsénico y Comisión Agua Segura. (noviembre, 2013). Informe a la Sala Constitucional: Avance respecto a la Atención de Sistemas de Acueducto referido a la presencia de Arsénico sobre la Norma Nacional. San José: Ministerio de Salud e Instituto Costarricense de Acueductos y Alcantarillados.

Herrera, R. et al. (abril, 2014). Clinical Characteristics of Chronic Kidney Disease of Nontraditional Causes in Salvadoren Farming Communities. Medicc Review, 16(2).

Instituto Tecnológico de Costa Rica. (2008). Atlas Digital Costa Rica 2008. Cartago: ITCR.

Jayasinglee, S. (abril, 2014). Chronic Kidney Disease of Unknown Etiology Should Be Renamed Chronic Agrochemical Nephropathy. Medicc Review, 16(2).

López, L. MD MS y colaboradores. (abril, 2014). Histopathology of Chronic Kidney Disease of Unknown Etiology in Salvadoran Agricultural Commuties. Medicc Review, 16(2).

Ministerio de Salud de Chile. (2010). Guía Clínica. Prevención de Enfermedad Renal Crónica. Santiago: MINSAL.

Mora, D. (2011). Informe sobre el estado de la Problemática de Arsénico en Costa Rica. Laboratorio Nacional de Aguas.

Mora, D. (2013). (octubre-diciembre, 2013). Agua para Consumo: Comparación de la Contaminación con Arsénico en Argentina, Chile y Centroamérica 2013. Revista del Colegio de Microbiólogos y Químicos Clínicos de Costa Rica ISSN: 2215-3713.

Mora. D. (2014). La problemática del arsénico. Costa Rica en el Contexto Latinoamericano. Laboratorio Nacional de Aguas; 2014.

National Kidney Foundation. (2009). Clinical Practice Guidelines for chronic kidney disease: evaluation, classification and stratification. Am. J. Kidney Dis. 39 (suppl 1), S 1-5266.

OMS/OPS. (septiembre-octubre, 2013). 52 ${ }^{\text {a }}$ Consejo Directivo 65 . Sesión del Comité Regional sobre La enfermedad Renal Crónica en Comunidades Agrícolas de Centroamérica. Washington, D.C.

Poder Ejecutivo de Costa Rica. (3 mayo, 2005). Reglamento para la Calidad del Agua Potable. Decreto Ejecutivo 32327, La Gaceta 84.

Ramírez Rubio, A. \& Kangsen Scammell, M. (2011). Enfermedad Renal Crónica en Nicaragua. Análisis Cualitativo de Entrevistas Semiestructurales con Médicos y Farmacéuticos. Universidad de Boston.

Rodríguez, M. (abril, 2014). Chronic Kidney Disease in Our Farming Communities Implications of on Epidemic. Medicc Review, 16(2).

Soyibo, A. \& Roberts, L. (2011). La enfermedad renal crónica en el Caribe. West Indian Med J., 60(4), 464.

Wanigasuriya, K. (abril, 2014). Update on Uncertain Etiology of Chronic Kidney Disease in Sri-Lanka North-Central Dry Zone. Medicc Review, 16(2).

Xavier F. et al. (abril, 2014). Chronic Kidney Disease and Associated Risk Factors in two Salvadoran Farming Communities, 2012. Medicc Review, 16(2). 